\title{
Study on Carbon - Oxygen Catalytic Combustion Mechanism based on Density Functional Theory
}

\author{
Yakun Qu', Xiaoguang Zhao', Yi Zhao' ${ }^{1}$ Zhenyu Dai ${ }^{1}$, Han Zhou' ${ }^{1}$, Jun Long ${ }^{1}$ \\ ${ }^{1}$ Research Institute of Petroleum Processing, SINOPEC, Beijing, 100083
}

KEYWORDS: Density Functional Theory; Catalyzer; Catalytic Combustion Mechanism

\begin{abstract}
Catalytic combustion is an important technique for utilization of fossil fuels, and the research on catalytic combustion mechanism could provide theoretical basis for the selection of catalyzer.In this paper, we adopt the Ni catalyst to promote the oxidation combustion reaction, and carry out study on carbon oxygen catalytic combustion mechanism based on density functional theory. The result suggests that $\mathrm{Ni}$ catalyst could significantly improve the carbon - oxygen catalytic combustion performance.
\end{abstract}

\section{Density Functional Theory Overview}

Density functional theory (DFT) is an expression form of quantum theory, which is developed based on Thomas-Fermi theory in 1960s. The wave function is seen as the basic physical systemin in traditional quantum theory, while density functional theory describes the physical properties of the ground state of the system by particle density, that is, the nature of the system is uniquely identified by its electron density distribution $(\rho)$.

Like Hartree-Fock method, DFT also introduce three approximations: Born-Oppenheimer approximation, adiabatic approximation and single-electron approximation. Adiabatic approximationseparates the motion of the nucleus from the motion of the electron; Hartree-Fockapproximation could simplify the multi- electronic problem to a single - electronic problem. DFT provides the theoretical basis for solving the problem, as it considers the exchange energy and the correlation energy, and adopts a variety of methods to reduce the error. As for Relativistic effect, DFT also makes some amendments, so that describe the multi-electron systemmore accurately.

The basic idea of DFT is to use the particle density function to describe the physical ground state atoms, molecules and solids properties. However, the theoretical basis wasnot really determined until Hohenberg-Kohn first and second theorems were proposed. Subsequently, Kohn and Sham's job made DFT a feasible theoretical method.

Hohenberg-Kohn first and second theorems have many forms of expression, but here briefly introduce the commonly-used one:

(1) Hohenberg-Kohn firsttheorem

For an $\mathrm{N}$ electronic system regardless ofspin that is in the outer potential field $\mathrm{V}(\mathrm{r})$, the outer potential field $\mathrm{V}(\mathrm{r})$ is uniquely identified by particle density $(\rho)$. This theorem shows that the ground state of the particle density in multi-electron system is corresponding to the external potential field. Meanwhile, it determines the number of electrons in the system, and then determines all properties of the system. This theorem lays a solid theoretical foundation for the DFT.

(2) Hohenberg-Kohn second theorem

In case that total number of particles remains unchanged, the obtained ground state energy is equal to the minimum value of the energy functional. If density functional representation of system 
energy is known, we can derive the ground state density from the variational condition, so that get all the physical properties of the ground state of the system.

According to Hohenberg-Kohn theorem, the ground state energy and the ground state particle density function could be obtained by variations of density function by energy functional. To complete the single particle image, construct density functions with $\mathrm{N}$ single particle wavelet function $\varphi_{i}(r)$.

$$
\rho(r)=\sum_{i=1}^{N}\left|\varphi_{2}(r)\right|^{2}
$$

Thus, variations of $\rho(r)$ could be replaced by variations of $\varphi_{\mathrm{i}}(r)$, while lagrange multipliers replaced by $E_{i}$. So we can obtain single-electron equation as follow:

$$
\left\{-\nabla^{2}+V_{K S}[\rho(r)]\right\} \varphi_{i}(r)=E_{i} \varphi_{i}(r)
$$

In the formula,

$$
V_{K S}[\rho(r)]=V_{e X t}(r)+V_{C}[\rho(r)]+V_{X C}[\rho(r)]=V_{e X t}(r)+d r^{\prime}\left|\frac{\rho\left(r^{\prime}\right)}{r-r^{\prime}}\right|+\frac{\delta E_{X C}[\rho]}{\delta \rho}
$$

The above three formulas constitute Kohn-Sham equation. The core of Kohn-Sham equation is to replace the particle Hamiltonian with interaction by the particle model without interaction, and then derive the single-electron equation. Similar to Hartree-Fockapproximation, in DFT, single-electron Kohn-Sham equationderivation is strictly described.

\section{Ni Catalytic Combustion Mechanism}

Under the same conditions of combustion, adding of additives could obviously improve the combustion efficiency, e.g. $\mathrm{H}_{2}$ /Arplasma pyrolysis testsimulates the rapid thermal decomposition of pulverized coal in raceway. Metal catalysts are recognized as the catalysts that have combustion catalyst effect. Ni catalyst plays aimportant role in reduce the ignition point of carbon-oxygen system, because it can effectively promote the interface reaction of carbon and oxygen, and it has high catalytic activity.However, it is easy to cause erosion of combustion equipment, meanwhile.

Composite catalyst is to mix two or more combustion supporting agents that have strengthened catalysis effect. In this study, we adopt the $\mathrm{Ni}-\mathrm{Al}_{2} \mathrm{O}_{3}$ composite catalyst to promote the oxidation combustion reaction. The influence of nickel powder on carbon-oxygen catalytic combustion reaction is shown in Table 1.

Table 1. Influence of nickel powder on carbon-oxygen catalytic combustion reaction

\begin{tabular}{|c|c|c|c|c|c|c|}
\hline \multirow{2}{*}{ No. } & \multirow{2}{*}{ Component } & \multicolumn{2}{|c|}{ Burn rate at different pressure (MPa) $\mathrm{r} / \mathrm{mm}^{-s^{-1}}$} & \multirow{2}{*}{$\mathrm{n}$} \\
\cline { 3 - 6 } & & 9 & 12 & 15 & 18 & \\
\hline 1 & Pure $\mathrm{Al}_{2} \mathrm{O}_{3}$ & 9.374 & 10.77 & 11.98 & 13.53 & 0.53 \\
\hline 2 & $2 \% \mathrm{Ni}$ mixture & 9.967 & 11.38 & 12.83 & - & 0.49 \\
\hline 3 & $3 \%$ Ni mixture & 9.969 & 11.48 & 12.96 & 13.32 & 0.42 \\
\hline
\end{tabular}


From Table 1 we can see that, adding of nickel powder in catalyzer could significantly affect the combustion performance. When adding $3 \% \mathrm{Ni}$ in the mixture, the burning rate pressure index would be decreased from 0.53 to 0.42 , comparing to pure $\mathrm{Al}_{2} \mathrm{O}_{3}$ catalyzer.

The catalytic mechanism of $\mathrm{Ni}$ in Carbon - Oxygen reaction should focus on adsorption of oxygen molecules on $\mathrm{Ni}$ surface. Therefore, the first step in the catalytic process may be $\mathrm{O}_{2-}$ moleculestably adsorbing on the surface of $\mathrm{Ni}$ and generating activated oxygen species. Only in this way could Nitransfer the adsorbed oxygen species to the carbon surface. According to the above speculation, we divide the reaction process of Ni catalytic carbon - oxygen oxidation into three parts: (1) generation of Nisurface active oxygen species; (2) interaction between carbon andNisurface active oxygen species; (3)reaction product removed from the Ni surface

(1) Generation of Nisurface active oxygen species

The configurations of $\mathrm{O}_{2}$ molecule adsorbing on four different locations on the clean surface of Ni(111)-p $(3 \times 3 \times 4)$ are shown as Figure 1, and the adsorption configuration parameters are shown in Table 2:

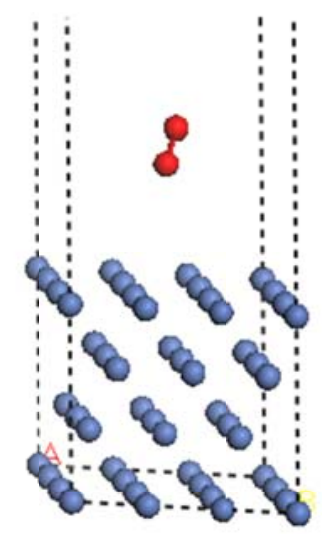

(a)

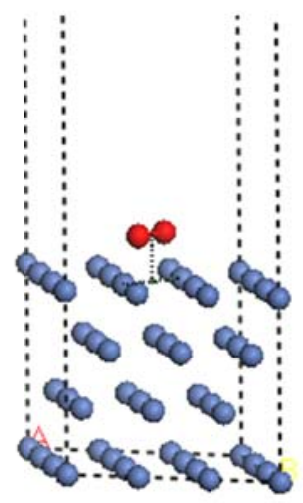

(c)

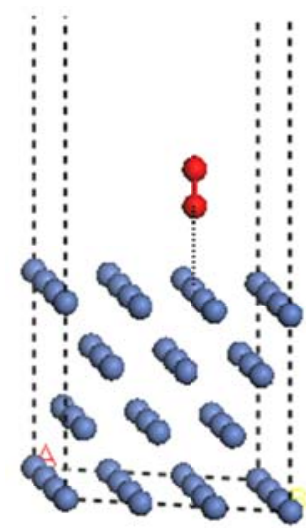

(b)

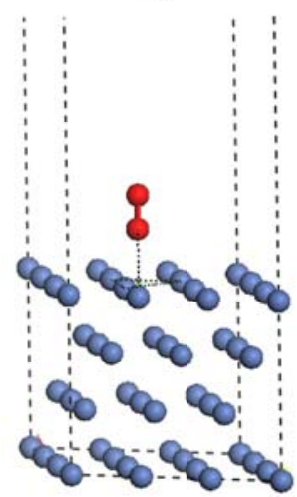

(d)

Figure 1. Physical adsorption configuration of $\mathrm{O} 2$ on Ni(111), a) the $\mathrm{O} 2$ adsorbed on the top of Ni atom, (b) the $\mathrm{O} 2$ adsorbed on the top of Ni-Nibridge, (d) the $\mathrm{O} 2$ adsorbed on the top hollow

Table 2. Adsorption energy, distance between $\mathrm{O}_{2}$ and surface atom, and distance between $\mathrm{O}-\mathrm{O}$ bonds for $\mathrm{O}_{2}$ adsorption on $\mathrm{Ni}(111)$ surface

\begin{tabular}{|c|c|c|c|}
\hline Model & $\mathrm{E}_{\mathrm{b}} / \mathrm{eV}$ & $\mathrm{d} / \mathrm{A}$ & $\mathrm{d}_{\text {O-o }} / \mathrm{A}$ \\
\hline Ni top & Unstable & 2.518 & 1.242 \\
\hline Bridge & 1.12 & 1.544 & 1.373 \\
\hline Hollow top & Unstable & 2.251 & 1.236 \\
\hline
\end{tabular}


From Table 2 we can see that, the adsorption configuration of $\mathrm{O}_{2}$ on $\mathrm{Ni}-\mathrm{Ni}_{\text {bridge }}$ is the most stable, which shows that the diffusion energy barrier of $\mathrm{O}_{2}$ on this surface is relatively high, so it not easy to diffuse.

In order to analyze the bonding mechanism of $\mathrm{O}_{2}$ adsorbed on the catalyst surface, we calculate the molecular density curve (DOS) of free $\mathrm{O}_{2}$ and adsorbed $\mathrm{O}_{2}$, as shown in Figure 2.

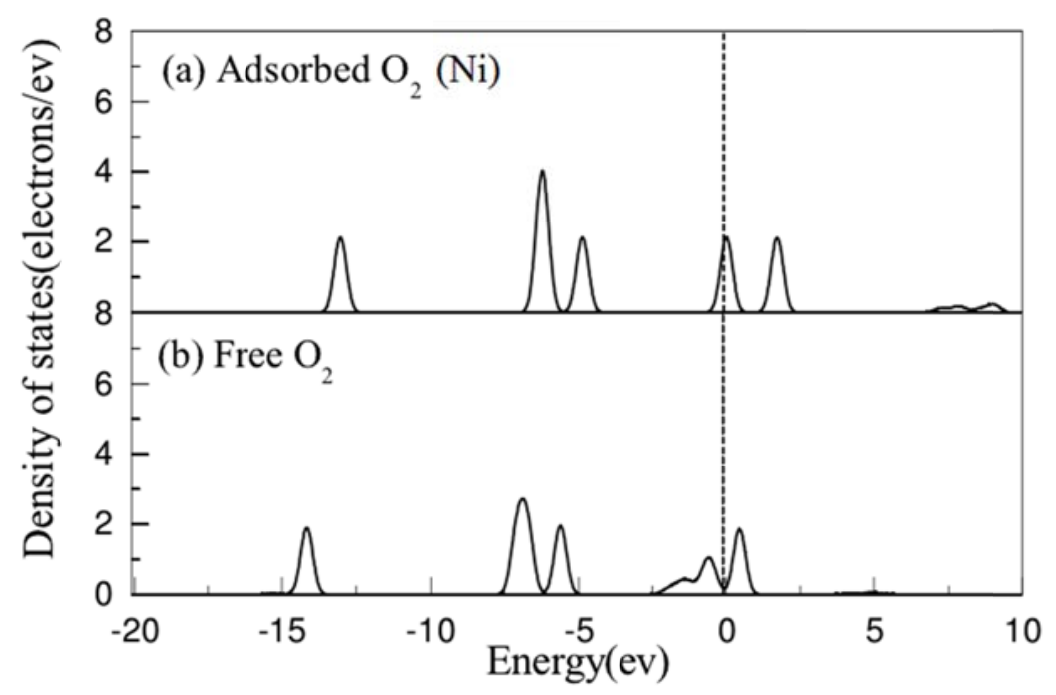

Figure 2. Molecular density curve of free $\mathrm{O} 2$ and adsorbed $\mathrm{O} 2$

The results shows that $\mathrm{Ni}(111)$ surface adsorption mode is exothermic reaction, and adsorption energy is large thanenergy in simple electronic reaction, so we can speculate that there is electron transfer between $\mathrm{Ni}$ and $\mathrm{O}_{2}$. Oxygen molecules could stably adsorb in $\mathrm{Ni}-\mathrm{Ni}$ bridges on the surface of $\mathrm{Ni}(111)$ and be activated, which provides reactive oxygen species for the subsequent reaction with carbon. This process can be expressed as:

$$
\mathrm{O}_{2}+\mathrm{Ni} \rightarrow \mathrm{NiO}_{x} \text { (peroxo) }
$$

(2) Interaction between carbon andNisurface active oxygen species

The configurations before and after interaction between $\mathrm{C}_{4}$ cluster and $\mathrm{Ni}(111)$ surface adsorbing oxygen molecule are shown in Figure 3.

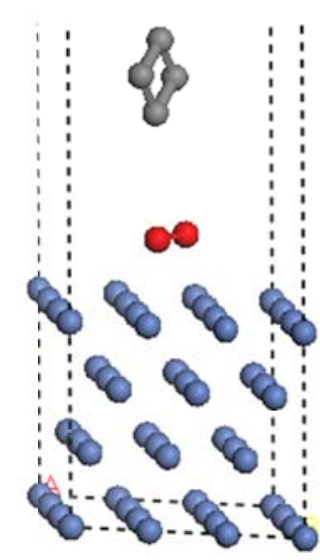

(a)

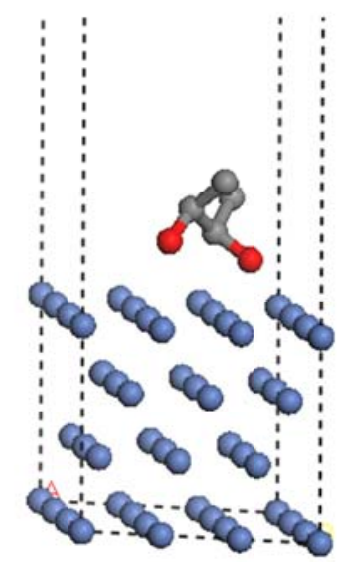

(b)

Figure 3. Adsorption configuration of a $\mathrm{C} 4$ cluster on the $\mathrm{O} 2 / \mathrm{Ni}$ surface, (a) before adsorbed, (b) after adsorbed 
Be comparing the two configurations, after $\mathrm{C}_{4}$ clusteradsorption, the structure appears distorted: two adjacent carbon atoms are bonded to the active oxygen atom, respectively; O-Obond length is stretched and broken off. Binding energy of this configuration is calculated to be $3.479 \mathrm{eV}$. It suggeststhat this process is strong exothermic process, which can be spontanerous.

These results suggest that carbon clusters can be oxidized in $\mathrm{O}_{2} / \mathrm{Ni}$ surface, and the process can be expressed as:

$$
\mathrm{C}_{\mathrm{n}}+\mathrm{NiO}_{\mathrm{x}}(\text { peroxo }) \rightarrow \mathrm{C}_{\mathrm{n}} \mathrm{O}_{\mathrm{x}}+\mathrm{Ni}
$$

(3) Reaction product desorbing from the Ni surface

In the above reaction configuration, $\mathrm{C}_{\mathrm{n}} \mathrm{Ox}$ or $\mathrm{CO}_{2}$ will be removed from the Ni surface under certain conditions. The possible configurations of the reaction products desorption from the surface of Ni are shown in Figure 4.

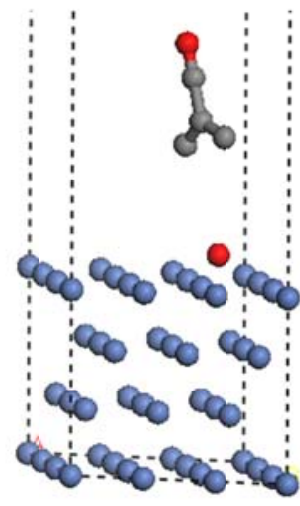

(a)

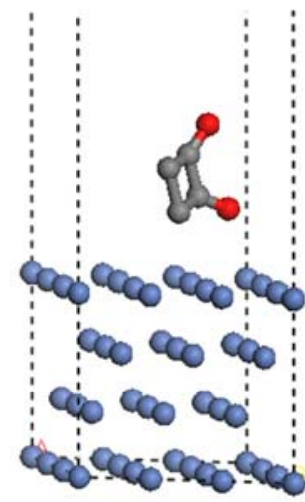

(b)

Figure 4. Possible configuration of product desorption from Ni surface, (a) Ni_O+C4O, (b)

$$
\mathrm{Ni}+\mathrm{C} 4 \mathrm{O} 2
$$

From the figure we can see that, the $\mathrm{Ni}$ surface configurations after desorption from $\mathrm{C}_{4} \mathrm{O}$ and $\mathrm{C}_{4} \mathrm{O}_{2}$ has a great change. Calculating the energy configuration, it shows that the structural energy after adsorption is larger than that before desorption, so it is endothermic process. Through transition state method, we calculate the configuration and energy barrier of transition states, as shown in Figure 5.

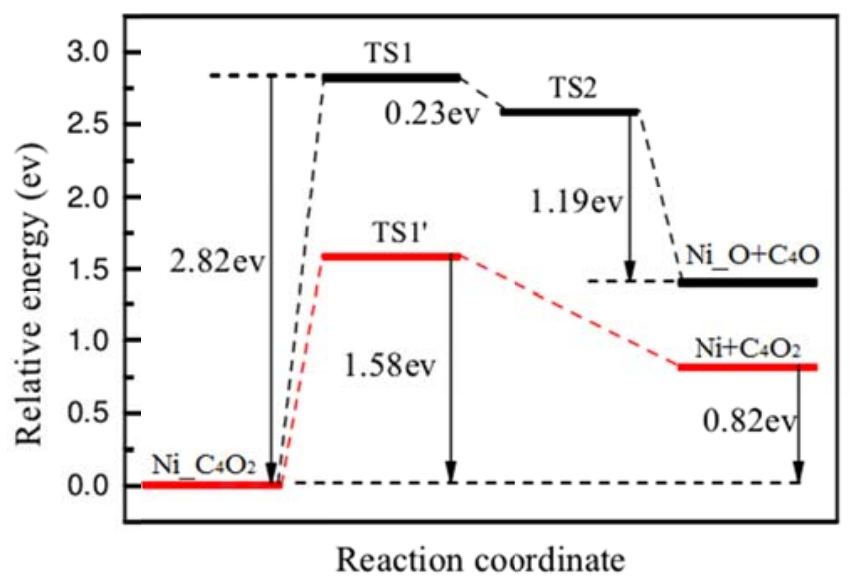

Figure 5. Potential energy diagram for different pathways of desorption of products on Ni surface 
Figure 5 shows that it requires to absorb $1.400 \mathrm{eV}$ when $\mathrm{C}_{4} \mathrm{O}$ desorbs from $\mathrm{Ni}_{-} \mathrm{C}_{4} \mathrm{O}_{2}$, and needs to cross the two energy barriers of $2.82 \mathrm{eV}$ and $2.59 \mathrm{eV}$; while it just requires to absorb $0.82 \mathrm{eV}$ when desorbs as the form of $\mathrm{C}_{4} \mathrm{O}_{2}$, and the energy barrier is as low as $1.58 \mathrm{eV}$.

\section{Conclusion}

Based on density functional theory, we have carried out systematic study on the process mechanism that the $\mathrm{Ni}-\mathrm{Al}_{2} \mathrm{O}_{3}$ composite catalyzer promoting oxygen transfer in carbon-oxygen combustion reaction, and the results shows that: 1) adding of nickel powder in catalyzer could significantly affect the combustion performance; 2) the $\mathrm{Ni}-\mathrm{Ni}$ bride bond on surface of $\mathrm{Ni}(111)$ is the active site of the catalytic reaction, $\mathrm{O}_{2}$ can adsorb and generate reactive oxygen species in these location; 3) $\mathrm{C}$ or $\mathrm{CO}$ is preferentially associated with the generation of reactive oxygen species, and the dissociation of the product is easy to occur. This study has explored the catalytic reaction path of $\mathrm{Ni}-\mathrm{Al}_{2} \mathrm{O}_{3}$ composite catalyzer in carbon-oxygen system, and explained the oxygen transfer / release mechanism in catalytic combustion process.

\section{Reference:}

[1] Liao S, Ying W, Fang D, et al. Kinetics of catalytic combustion in air over Pt/A12O3/A1 catalyst[J]. Reaction Kinetics \& Catalysis Letters, 2005, 85(1):205-213.

[2] Chen M, Wang X, Jiao W L, et al. Research Progress in Mechanism and Catalysts for Methane Catalytic Combustion[J]. Gas \& Heat, 2010.

[3] Zhi-Rong L I, Zhao J. Probe into the Mechanism of the Catalytic Combustion Technique and Its Research Progress[J]. Sci/tech Information Development \& Economy, 2005.

[4] Parr R G. Density Functional Theory[J]. Chemical \& Engineering News, 1990, 68(1).

[5] Ansari A, Rajaraman G. Dataset for Modelling Reaction Mechanisms Using Density Functional Theory: Mechanism of ortho-Hydroxylation by High-Valent Iron-Oxo Species[J]. Dataset Papers in Science, 2014, 2014:1-7. 